\title{
Nonlocal gravity and gravitational-wave observations
}

\section{Enis Belgacem, ${ }^{a}$ Yves Dirian, ${ }^{b}$ Andreas Finke, ${ }^{a}$ Stefano Foffa, ${ }^{a}$ Michele Maggiore, ${ }^{a}$}

\author{
${ }^{a}$ Département de Physique Théorique and Center for Astroparticle Physics, \\ Université de Genève, 24 quai Ansermet, CH-1211 Genève 4, Switzerland \\ ${ }^{b}$ Center for Theoretical Astrophysics and Cosmology, Institute for Computational Science, \\ University of Zürich, CH-8057 Zürich, Switzerland
}

\begin{abstract}
We discuss a modified gravity model which fits cosmological observations at a level statistically indistinguishable from $\Lambda \mathrm{CDM}$ and at the same time predicts very large deviations from General Relativity (GR) in the propagation of gravitational waves (GWs) across cosmological distances. The model is a variant of the RT nonlocal model proposed and developed by our group, with initial conditions set during inflation, and predicts a GW luminosity distance that, at the redshifts accessible to LISA or to a third-generation GW detector such as the Einstein Telescope (ET), can differ from that in GR by as much as $60 \%$. An effect of this size could be detected with just a single standard siren with counterpart by LISA or ET. At the redshifts accessible to a LIGO/Virgo/Kagra network at target sensitivity the effect is smaller but still potentially detectable. Indeed, for the recently announced LIGO/Virgo NS-BH candidate S190814bv, the RT model predicts that, given the measured GW luminosity distance, the actual luminosity distance, and the redshift of an electromagnetic counterpart, would be smaller by as much as $7 \%$ with respect to the value inferred from $\Lambda$ CDM.
\end{abstract}




\section{Contents}

1 Introduction $\quad 1$

2 The RT nonlocal gravity model $\quad 2$

3 Detectability of the effect $\quad 6$

3.1 Sensitivity to $\Xi_{0}$ of $2 \mathrm{G}$ and $3 \mathrm{G}$ detectors 6

3.2 Application to the candidate NS-BH event S190814bv 7

\section{Introduction}

The search for deviations from $\Lambda \mathrm{CDM}$ and of modifications of gravity at cosmological scales has been a main theme of cosmological observations over the last decades. Modified gravity theories give rise to a different cosmological evolution for the background, usually encoded in the dark energy (DE) equation of state $w_{\mathrm{DE}}(z)$, and to different scalar and tensor cosmological perturbations. Observations indicate that, for the background evolution and scalar perturbations, deviations cannot exceed a few percent (although a most notable discrepancy exists between the local measurement of $H_{0}$ and the value inferred by Planck assuming $\Lambda$ CDM [1]). For instance, at the background level, using the simple parametrization $w_{\mathrm{DE}}(z)=w_{0}$, Planck 2015 combined with other datasets gives $w_{0}=-1.006 \pm 0.045$ [2], i.e. the deviation from $\Lambda \mathrm{CDM}$ is bounded at the $4.5 \%$ level. Similarly, the DES Y1 results [3] put bounds at the level of $7 \%$ on the deviation of scalar perturbations from $\Lambda$ CDM. Tensor perturbations, i.e. GWs propagating on a cosmological background, are instead still a rather virgin territory, that we are beginning to explore thanks to the extraordinary LIGO/Virgo observations [4-7].

In GR the propagation of GWs is governed by the equation

$$
\tilde{h}_{A}^{\prime \prime}+2 \mathcal{H} \tilde{h}_{A}^{\prime}+k^{2} \tilde{h}_{A}=0,
$$

where $A=\{+, \times\}, h^{\prime}=\partial_{\eta} h, \eta$ is conformal time, $\mathcal{H}=a^{\prime} / a$ and $a(\eta)$ is the scale factor. In modified gravity this equation is in general different. However, a modification of the coefficient of the $k^{2}$ term gives a speed of GWs different from that of light, which is now excluded at a level $O\left(10^{-15}\right)$ [7], and indeed this has ruled out a large class of modifications of GR [8-11]. Still, in many modified gravity theories GW propagation is governed by the equation

$$
\tilde{h}_{A}^{\prime \prime}+2 \mathcal{H}[1-\delta(\eta)] \tilde{h}_{A}^{\prime}+k^{2} \tilde{h}_{A}=0,
$$

with some function $\delta(\eta)$ [12-23]. An important consequence of this modification is that, in the waveform of a coalescing binary, the factor $1 / d_{L}^{\mathrm{em}}(z)$, where $d_{L}^{\mathrm{em}}(z)$ is the standard luminosity distance measured by electromagnetic probes, is replaced by a 'GW luminosity' distance $d_{L}^{\mathrm{gw}}(z)$ (similar effects take place in theories with extra dimensions [24, 25]). The relation between $d_{L}^{\mathrm{gw}}(z)$ and $d_{L}^{\mathrm{em}}(z)$ is [17]

$$
d_{L}^{\mathrm{gw}}(z)=d_{L}^{\mathrm{em}}(z) \exp \left\{-\int_{0}^{z} \frac{d z^{\prime}}{1+z^{\prime}} \delta\left(z^{\prime}\right)\right\} .
$$


For comparing with observations it is useful to have a parametrization of the effect in terms of a small number of parameters, rather than a full function $\delta(z)$. A very convenient parametrization, in terms of two parameters $\left(\Xi_{0}, n\right)$, has been proposed in [19],

$$
\frac{d_{L}^{\mathrm{gw}}(z)}{d_{L}^{\mathrm{em}}(z)}=\Xi_{0}+\frac{1-\Xi_{0}}{(1+z)^{n}} .
$$

This parametrization reproduces the fact that, as $z \rightarrow 0, d_{L}^{\mathrm{gw}} / d_{L}^{\mathrm{em}} \rightarrow 1$ since, as the redshift of the source goes to zero, there can be no effect from modified propagation. In the limit of large redshifts, in eq. (1.4) $d_{L}^{\mathrm{gw}} / d_{L}^{\mathrm{em}}$ goes to a constant value $\Xi_{0}$. This is motivated by the fact that in typical DE models the deviations from GR only appear in the recent cosmological epoch, so $\delta(z)$ goes to zero at large redshift and, from eq. $(1.3), d_{L}^{\mathrm{gw}}(z) / d_{L}^{\mathrm{em}}(z)$ saturates to a constant. Indeed, in ref. [23] have been worked out the predictions of some of the beststudied modified gravity models such as several examples of Horndeski and DHOST theories, non-local infrared modifications of gravity, or bigravity theories. It has been found that all these models (except bigravity, where there are non-trivial oscillations due to the interaction between the two metrics) predict a propagation equation of the form (1.2), with functions $\delta(z)$ such that $d_{L}^{\mathrm{gw}}(z) / d_{L}^{\mathrm{em}}(z)$ is very well fitted by eq. (1.4).

The parametrization (1.4) has been used in $[19,26]$ to forecast the sensitivity to modified GW propagation of a network of detectors composed by advanced LIGO/Virgo/Kagra (HLVKI) at their target sensitivity, and of a third generation detectors such as the Einstein Telescope (ET), using as standard sirens the coalescence of binary neutron stars (BNS) with an observed electromagnetic counterpart. Using state-of-the-art mock catalogs for the GW events and for the detection of an associated GRB, it is estimated that the HLVKI network can reach an accuracy on $\Xi_{0}$ of order $20 \%$ over a few years of data taking, while ET could measure it to about $1 \%$. In [23] has been studied the sensitivity of LISA, using as standard sirens the coalescence of supermassive black holes $(\mathrm{SMBH})$, and it has been found that $\Xi_{0}$ could be measured to $(1-4) \%$ accuracy, depending on assumptions on the population of SMBH black hole binaries.

The crucial question is therefore what is the size of the deviations from the GR value $\Xi_{0}=1$, that one could expect from viable modified gravity models. As mentioned above, for such models the deviations from $\Lambda$ CDM are bounded at the level of $(4-5) \%$ for the background evolution and about $7 \%$ for scalar perturbations, so one might expect that the deviations in the tensor sector will be of the same order. However, below we will present a model that is fully viable, and which nevertheless can give deviations as large as $60 \%$ in the tensor sector. This is excellent news for GW detectors, since it means that the new window on the Universe which is being opened by GW observations might reserve surprises from the point of view of cosmology, to the extent that advanced GW detectors could become the best instruments for detecting deviations from GR at cosmological scales.

\section{The RT nonlocal gravity model}

Among the plethora of existing modified gravity models, a class that has been much developed in the last few years are nonlocal infrared modifications of gravity. The underlying idea, that in different forms goes back to old works [27-31], is that quantum gravity at large distances could induce cosmological effects, related to the emergence of IR divergences in spacetimes of cosmological interest such as de Sitter space. These quantum effects generate nonlocal terms in the quantum effective action. This could lead to the appearance of terms involving the 
inverse of the d'Alembertian operator, $\square^{-1}$, that are relevant in the infrared and therefore affect the cosmological evolution. A first-principle understanding of these infrared effects is currently very difficult, so the work in this direction has been mostly of phenomenological nature, trying to identify models with interesting cosmological properties. However, already building a viable model turns out to be highly nontrivial. A nonlocal gravity model based on this idea was proposed in [32] (see also [33] for earlier work), but is now ruled out [34]. A more recent twist of the idea is that infrared effects might generate dynamically a mass, associated to nonlocal terms. The first viable model of this type was proposed in [35], elaborating on earlier work in [36, 37], and is defined by the nonlocal equation of motion

$$
G_{\mu \nu}-\left(m^{2} / 3\right)\left(g_{\mu \nu} \square^{-1} R\right)^{\mathrm{T}}=8 \pi G T_{\mu \nu} .
$$

Here $m$ is a mass scale, eventually taken of order $H_{0}$, that replaces the cosmological constant, and the superscript ' $\mathrm{T}$ ' denotes the extraction of the transverse part of a tensor, based on the fact that any symmetric tensor $S_{\mu \nu}$ can be decomposed as $S_{\mu \nu}=S_{\mu \nu}^{\mathrm{T}}+(1 / 2)\left(\nabla_{\mu} S_{\nu}+\nabla_{\nu} S_{\mu}\right)$, where the transverse part $S_{\mu \nu}^{\mathrm{T}}$ satisfies $\nabla^{\mu} S_{\mu \nu}^{\mathrm{T}}=0$. We refer to it as the 'RT' model, where R stands for the Ricci scalar and T for the extraction of the transverse part of $S_{\mu \nu} \equiv g_{\mu \nu} \square^{-1} R$. Detailed discussions of the reasoning that led to this specific structure (that corresponds to a dynamical mass generation for the conformal mode), of conceptual aspects related to the appearance of these nonlocal terms (that respect causality and do not introduce extra degrees of freedom), and of its cosmological consequences can be found in the reviews [38, 39]. Here we limit ourselves to recalling that several studies [34, 35, 39-48] have shown that the RT model has a viable cosmological background evolution, where the nonlocal term acts as an effective dark energy density and drives accelerated expansion in the recent epoch; it has stable cosmological perturbations in the scalar and tensor sectors (a nontrivial condition that ruled out many modified gravity models); tensor perturbations propagate at the speed of light; the model fits $\mathrm{CMB}, \mathrm{BAO}, \mathrm{SNe}$ and structure formation at a level statistically equivalent to $\Lambda \mathrm{CDM}$; and it reproduces the successes of GR at solar system and laboratory scales. Studies of possible variations of the idea have singled out the RT model as the only known nonlocal model that passes all these tests.

A point that will be important in the following is that nonlocal models have a hidden freedom related to the choice of initial conditions for some auxiliary fields that are introduced to write the equations of motion in local form (or, equivalently, there is an implicit freedom in the definition of $\square^{-1}$ ). Once again, we refer the reader to $[35,38,39]$ for full discussions. However, the bottom line is that, if one had an explicit derivation of the effective nonlocal terms of the quantum effective action from the fundamental underlying local theory, one could derive these initial conditions explicitly in terms of those of the metric. In the absence of such a derivation, these initial conditions must be treated as phenomenological parameters. This, however, does not introduce as much freedom as one might fear. Indeed, if we set initial conditions $\mathcal{O}(1)$ during radiation dominance (RD), the evolution is fully determined because the freedom in the initial conditions either corresponds to re-introducing a cosmological constant, that we set to zero because, at least in the minimal setting, we want to reproduce the accelerated expansion without reintroducing a cosmological constant, or else corresponds to irrelevant directions in parameter space (the same happens at the level of cosmological perturbations). The situation is different if we start the evolution in a primordial inflationary phase; in this case, because of the presence of a growing mode, if we start with initial conditions of order one at some time when there are still $\Delta N$ e-folds until the end of inflation, an auxiliary field gets a value of order $e^{\alpha \Delta N}$ when inflation ends and RD begins, with $\alpha$ a 


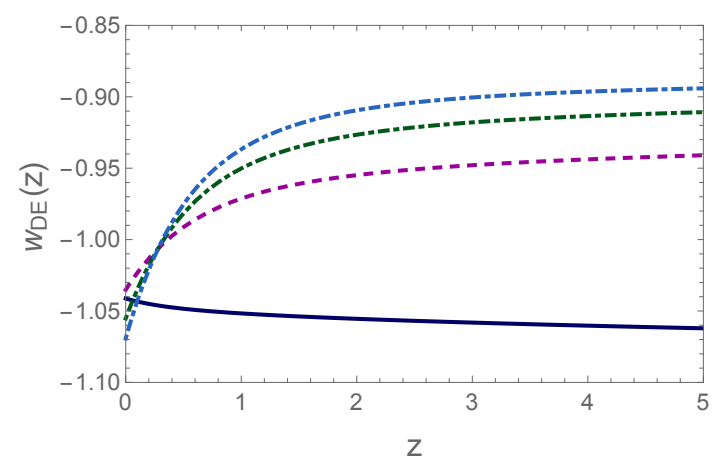

Figure 1. The DE equation of state $w_{\mathrm{DE}}(z)$ as a function of redshift for the minimal RT model (blue solid line), for $\Delta N=34$ (magenta, dashed), $\Delta N=50$ (green, dot-dashed) and $\Delta N=64$ (cyan, dot-dashed).

known constant. This instability has no effect on the evolution during inflation (neither at the background level not for the perturbations), since the energy scale associated to the nonlocal term is negligible with respect to the inflationary scale. However, the subsequent evolution during RD inherits a dependence on $\Delta N$. Full details are given in [38, 49]. The model that we study below is indeed the RT model with initial conditions set during inflation. For inflation taking place at a scale $M$, assuming instantaneous reheating, the minimum number of efolds required to solve the flatness and horizon problems is

$$
\Delta N \simeq 64-\log \frac{10^{16} \mathrm{GeV}}{M} .
$$

In the following, beside the 'minimal' model defined by initial conditions of order one during $\mathrm{RD}$, which corresponds to $\Delta N=0$, we will study also the cases $\Delta N=\{34,50,64\}$ that, in the above approximation, correspond to $M=\left\{10^{3}, 10^{10}, 10^{16}\right\} \mathrm{GeV}$, respectively. The result for the background evolution, i.e. for $w_{\mathrm{DE}}(z)$, were already given in [38], and we reproduce them in Fig. 1. In the minimal model $w_{\mathrm{DE}}$ is always on the phantom side, $w_{\mathrm{DE}}(z)<-1$, while for large $\Delta N$ it evolves from a non-phantom value at large $z$ toward a phantom value today, with phantom crossing near $z \simeq 0.30-0.35$. In any case at $z<1$, when DE becomes important, all these curves are within about $5 \%$ of the $\Lambda$ CDM value -1 , so the background evolution of these models is still quite close to that of $\Lambda \mathrm{CDM}$.

We then study whether this evolution, together with the associated scalar perturbations, is consistent with observations. Cosmological perturbations for the RT model were computed in [44] and, for the minimal model, were implemented into a Markov Chain Monte Carlo (MCMC) and compared with data in [46, 47]. We extend here the analysis to $\Delta N=34,50,64$. We use the same datasets as in our previous works, namely the 2015 Planck data, JLA supernovae and a compilation of BAO, and we vary the standard set of cosmological parameters (see [39] for details), including the sum of neutrino masses. The results are shown in Table 1 . From the mean values of the parameters we see that the RT model is very close to $\nu \Lambda \mathrm{CDM}$ (i.e. $\Lambda \mathrm{CDM}$ where the neutrino masses are allowed to vary), particularly for large $\Delta N$. In the last line we give the difference of the $\chi^{2}$ of a given model, with respect to $\nu \Lambda$ CDM. According to the conventional Jeffreys' scale, a difference $\left|\Delta \chi^{2}\right| \leq 2$ implies statistically equivalence between the two models compared, while $\left|\Delta \chi^{2}\right| \gtrsim 2$ suggests "weak evidence", and $\left|\Delta \chi^{2}\right| \gtrsim 6$ indicates "strong evidence". Thus, all models shown in Table 1 fit the data at a statistically equivalent level. Observe also that the RT model does 


\begin{tabular}{|l||c|c|c|c|c|}
\hline Parameter & $\nu \Lambda \mathrm{CDM}$ & $\mathrm{RT}$, minimal & $\mathrm{RT}, \Delta N=34$ & $\mathrm{RT}, \Delta N=50$ & $\mathrm{RT}, \Delta N=64$ \\
\hline$H_{0}$ & $67.60_{-0.55}^{+0.66}$ & $68.35_{-0.71}^{+0.75}$ & $67.68_{-0.59}^{+0.67}$ & $67.71_{-0.62}^{+0.56}$ & $67.66_{-0.64}^{+0.68}$ \\
$\sum_{\nu} m_{\nu}[\mathrm{eV}]$ & $<0.10($ at $1 \sigma)$ & $0.126_{-0.101}^{+0.055}$ & $<0.10($ at $1 \sigma)$ & $<0.08($ at $1 \sigma)$ & $<0.09$ (at $1 \sigma)$ \\
$\omega_{c}$ & $0.1189_{-0.0011}^{+0.0011}$ & $0.1194_{-0.0012}^{+0.0012}$ & $0.1186_{-0.0012}^{+0.0012}$ & $0.1185_{-0.0011}^{+0.0012}$ & $0.1184_{-0.0012}^{+0.0012}$ \\
$100 \omega_{b}$ & $2.229_{-0.015}^{+0.014}$ & $2.225_{-0.015}^{+0.015}$ & $2.230_{-0.014}^{+0.016}$ & $2.231_{-0.016}^{+0.015}$ & $2.232_{-0.016}^{+0.016}$ \\
$\ln \left(10^{10} A_{s}\right)$ & $3.071_{-0.029}^{+0.026}$ & $3.070_{-0.032}^{+0.029}$ & $3.076_{-0.031}^{+0.027}$ & $3.075_{-0.028}^{+0.027}$ & $3.080_{-0.029}^{+0.028}$ \\
$n_{s}$ & $0.9661_{-0.0043}^{+0.0043}$ & $0.9648_{-0.0043}^{+0.0045}$ & $0.9670_{-0.0045}^{+0.0045}$ & $0.9670_{-0.0046}^{+0.0042}$ & $0.9673_{-0.0048}^{+0.0046}$ \\
$\tau_{\mathrm{re}}$ & $0.06965_{-0.01549}^{+0.01393}$ & $0.06858_{-0.01721}^{+0.01534}$ & $0.07257_{-0.01585}^{+0.01491}$ & $0.07183_{-0.01518}^{+0.01430}$ & $0.07462_{-0.01609}^{+0.01488}$ \\
\hline$\Omega_{M}$ & $0.3109_{-0.0084}^{+0.0069}$ & $0.3061_{-0.0091}^{+0.0079}$ & $0.3095_{-0.0081}^{+0.0077}$ & $0.3087_{-0.0074}^{+0.0075}$ & $0.3091_{-0.0086}^{+0.0077}$ \\
$z_{\mathrm{re}}$ & $9.150_{-1.355}^{+1.396}$ & $9.058_{-1.487}^{+1.587}$ & $9.417_{-1.376}^{+1.429}$ & $9.349_{-1.279}^{+1.402}$ & $9.604_{-1.467}^{+1.402}$ \\
$\sigma_{8}$ & $0.8157_{-0.0104}^{+0.0135}$ & $0.8196_{-0.0130}^{+0.0165}$ & $0.8162_{-0.0112}^{+0.0140}$ & $0.8164_{-0.0112}^{+0.0128}$ & $0.8166_{-0.0114}^{+0.0129}$ \\
\hline$\Delta \chi^{2}$ & 0 & 0.76 & -1.10 & -1.20 & -1.18 \\
\hline
\end{tabular}

Table 1. Mean values (with $1 \sigma$ errors) of the parameters for $\nu \Lambda \mathrm{CDM}$ and the RT model with $\Delta N=0,34,50,64$, using $\mathrm{CMB}, \mathrm{BAO}$ and SNe. $H_{0}$ is in units of $\mathrm{km} \mathrm{s}^{-1} \mathrm{Mpc}^{-1}$. The last line gives the difference in the $\chi^{2}$ of each given model with respect to $\nu \Lambda \mathrm{CDM}$. The RT model with $\Delta N=50$ or with $\Delta N=64$ fit the data slightly better than $\nu \Lambda \mathrm{CDM}$, but the difference is not statistically significant.
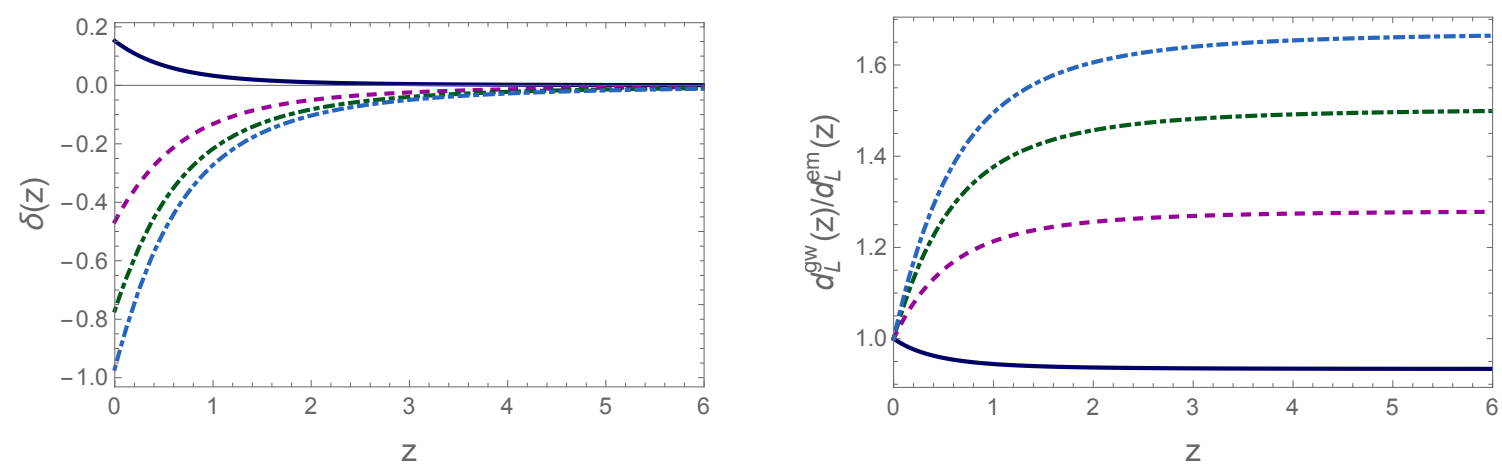

Figure 2. The functions $\delta(z)$ (left panel) and $d_{L}^{\mathrm{gw}}(z) / d_{L}^{\mathrm{em}}(z)$ (right panel) for the minimal RT model (blue solid line), for $\Delta N=34$ (magenta, dashed), $\Delta N=50$ (green, dot-dashed) and $\Delta N=64$ (cyan, dot-dashed).

not relieve the tension with the local $H_{0}$ measurement, particularly at large $\Delta N$. Indeed, recent work indicates that it might not be possible to solve the $H_{0}$ tension solely with a modification of the late-Universe dynamics [50, 51].

We next discuss tensor perturbations. The equation for tensor perturbations in the RT model is of the form (1.2), with $\delta(\eta)$ determined by the evolution of the auxiliary fields of the model [47]. Performing the numerical integration of the equations of motion we obtain $\delta(z)$, shown in the left panel of Fig. 2. Using eq. (1.3) we then obtain the ratio $d_{L}^{\mathrm{gw}}(z) / d_{L}^{\mathrm{em}}(z)$, which is shown in the right panel of Fig. 2 (the plot for the minimal model was already shown in [23]). The result is quite surprising: for large $\Delta N, d_{L}^{\mathrm{gw}}(z) / d_{L}^{\mathrm{em}}(z)$ becomes larger than one, corresponding to a smaller GW amplitude compared to the GR prediction. What 


\begin{tabular}{|l||c|c|c|c|}
\hline Parameter & RT, minimal & RT, $\Delta N=34$ & RT, $\Delta N=50$ & RT, $\Delta N=64$ \\
\hline$\Xi_{0}$ & 0.93 & 1.28 & 1.50 & 1.67 \\
$n$ & 2.59 & 2.07 & 1.99 & 1.94 \\
$\delta(0)$ & 0.15 & -0.47 & -0.77 & -0.97 \\
\hline
\end{tabular}

Table 2. The predictions for $\Xi_{0}, n$ and $\delta(z=0)$ of the RT model with $\Delta N=0,34,50,64$ keeping $\Omega_{M}$ and $H_{0}$ fixed at the values $\Omega_{M}=0.30$ and $H_{0}=68.80$ and varying only $\Delta N$.

\begin{tabular}{|l||c|c|c|c|}
\hline Parameter & RT, minimal & RT, $\Delta N=34$ & $\mathrm{RT}, \Delta N=50$ & $\mathrm{RT}, \Delta N=64$ \\
\hline$\Xi_{0}$ & 0.93 & 1.27 & 1.49 & 1.65 \\
$n$ & 2.59 & 2.08 & 2.00 & 1.95 \\
$\delta(0)$ & 0.15 & -0.46 & -0.76 & -0.95 \\
\hline
\end{tabular}

Table 3. As in Table 2, using for each $\Delta N$ the mean values for $\Omega_{M}$ and $H_{0}$ given in Table 1.

is most remarkable, however, is the size of the deviation from the GR value $\Xi_{0}=1$, that in the minimal model was about $6.6 \%$, while for $\Delta N=64$ is now at the level of $65 \%$, one order of magnitude larger! All the curves are perfectly fitted by eq. (1.4). Keeping $\Omega_{M}$ and $H_{0}$ fixed at the values $\Omega_{M}=0.30$ and $H_{0}=68.80$ and varying only $\Delta N$ we get the values shown in Table 2. If instead for each model we use its own mean values of the parameters from Table 1, we find the results shown in Table 3.

\section{$3 \quad$ Detectability of the effect}

\subsection{Sensitivity to $\Xi_{0}$ of $2 \mathrm{G}$ and $3 \mathrm{G}$ detectors}

The estimates of detector sensitivities to $\Xi_{0}$ mentioned above show that deviations from GR at this level are measurable at $3 \mathrm{G}$ detectors, and possibly even at $2 \mathrm{G}$ detectors. The analysis of ref. [26], using mock catalogs of joint GW-GRB detections and assuming the functional form (1.4), which fits perfectly the prediction of the RT model, shows that the HLVKI network in $10 \mathrm{yr}$ of run could measure $\Xi_{0}$ to an accuracy $\Delta \Xi_{0} \simeq 0.1$, while a shorter and more realistic time span, say 2-3 years, could still be enough to reach $\Delta \Xi_{0} \simeq 0.2$. This would be sufficient to detect with good confidence the difference between the prediction $\Xi_{0}=1.6$ of the $\Delta N=64$ scenario, and the GR value $\Xi_{0}=1$. Observe that in [26] were only studied the coincidences of GW events with GRBs. For well-localized events, several more counterparts could be obtained with optical/IR telescopes. Note that, at the redshifts accessible to the HLVKI network, we are not yet in the asymptotic regime $d_{L}^{\mathrm{gw}}(z) / d_{L}^{\mathrm{em}}(z) \simeq \Xi_{0}$, a fact that is anyhow taken into account by the use of the parametrization (1.4) in the analysis. Observe that being able to detect events at not-too-small redshifts is crucial here. In fact, to first order in $z$, eq. (1.3) becomes $d_{L}^{\mathrm{gw}}(z) / d_{L}^{\mathrm{em}}(z) \simeq 1-z \delta(0)$, so the deviation from 1 is linear in $z$. An event such as GW170817, at $z \simeq 0.01$, therefore gives a measure of $\delta(0)$ with an error 10 times larger than an event at $z=0.1$ with a comparable observational error. Since the overall error scales with the number of similar events roughly as $1 / \sqrt{N}$, a single event at $z=0.1$ contributes as much as $\mathcal{O}(100)$ events at $z=0.01$.

For a 3G detector such as ET the perspectives look extremely interesting. ET could see hundreds of BNS with counterpart to $z \simeq 2$, where $d_{L}^{\mathrm{gw}}(z) / d_{L}^{\mathrm{em}}(z) \simeq \Xi_{0}$, with an error $\Delta d_{L}^{\mathrm{gw}} / d_{L}^{\mathrm{gw}}$ of order $(5-10) \%$ (see sect. 2.3 .1 and Fig. 5 of [26]). With this sensitivity, already a single event with electromagnetic counterpart, or just a few of them, could be enough to 
discriminate between $\Xi_{0} \simeq 1.6$ and $\Xi_{0}=1$. With the few hundreds of joint GW-GRB detection estimated in [26], one can reach $\Delta \Xi_{0} / \Xi_{0} \simeq 1 \%$ or better.

Also for LISA the perspectives are quite exciting. From Table 2 of [23], LISA could measure $\Xi_{0}$ with an error $\Delta \Xi_{0} \simeq\{0.023,0.036,0.044\}$ in three different scenarios for SMBH formations that lead to catalogs containing, respectively, $N=\{32,12,9\}$ events. These numbers are well reproduced by $\Delta \Xi_{0} \simeq 0.13 / \sqrt{N}$, so each SMBH event gives a measure of $\Xi_{0}$ with an average accuracy of about $13 \%$ (which becomes $6 \%$ if we use a more optimistic scenario for the error on the redshift determination of the source). Thus, a single SMBH event at LISA could be sufficient to detect the effect.

It is remarkable that such a measurement would reveal the nature of dark energy, and at the same time would give information on primordial inflation through its dependence on $\Delta N$.

\subsection{Application to the candidate NS-BH event S190814bv}

It is very interesting to see how the above predictions apply to the very recent candidate event S190814bv, that, at the time of writing, has just been posted ${ }^{1}$ on the public database of GW candidate events of the LIGO/Virgo collaborations (see https://gracedb.ligo. org/superevents/S190814bv/). This event has an extremely low false alarm rate (less than one false alarm per $10^{25} \mathrm{yr}$ ), and is currently classified as a neutron-star - black-hole (NS-BH) coalescence with $>99 \%$ probability. At the time of writing, the information on the event has just been disseminated to the network of electromagnetic telescopes, and the search for an electromagnetic counterpart is under way. Even if information on this candidate event is still preliminary, still it is interesting to estimate the effect of modified GW propagation on an event of this type. Currently, the best estimate for the luminosity distance of the event, based on the GW signal, is $d_{L}=(267 \pm 52) \mathrm{Mpc}$. In $\Lambda \mathrm{CDM}$, this would be the same as the luminosity distance to the source measured by electromagnetic signals, while we have seen that in the RT nonlocal model (and more general, in basically all modified gravity models) there are two different notions of luminosity distance, the GW luminosity distance $d_{L}^{\mathrm{gw}}$, which is the quantity measured by GW detectors, and the 'electromagnetic' luminosity distance $d_{L}^{\mathrm{em}}$ measured by electromagnetic observations. In $\Lambda \mathrm{CDM}$, where the two notions coincide, we write simply $d_{L}^{\mathrm{gw}}(z)=d_{L}^{\mathrm{em}}(z) \equiv d_{L}(z)$. From a measurement of $d_{L}(z)$, the redshift of the source can be obtained by inverting the expression

$$
d_{L}(z)=\frac{1+z}{H_{0}} \int_{0}^{z} \frac{d \tilde{z}}{\sqrt{\Omega_{R}(1+\tilde{z})^{4}+\Omega_{M}(1+\tilde{z})^{3}+\Omega_{\Lambda}}},
$$

where $\Omega_{R}, \Omega_{M}$ and $\Omega_{\Lambda}=1-\Omega_{R}-\Omega_{M}$ are the density fractions associated to radiation, matter and to the cosmological constant, respectively. In contrast, in the RT model the electromagnetic luminosity distance is

$$
d_{L}^{\mathrm{em}}(z)=\frac{1+z}{H_{0}} \int_{0}^{z} \frac{d \tilde{z}}{\sqrt{\Omega_{R}(1+\tilde{z})^{4}+\Omega_{M}(1+\tilde{z})^{3}+\rho_{\mathrm{DE}}(\tilde{z}) / \rho_{0}}}
$$

where $\rho_{\mathrm{DE}}(z)$ is the dark energy density in the RT model and $\rho_{0}=3 H_{0}^{2} /(8 \pi G)$, and the GW luminosity distance is very well reproduced by

$$
d_{L}^{\mathrm{gw}}(z)=\left[\Xi_{0}+\frac{1-\Xi_{0}}{(1+z)^{n}}\right] d_{L}^{\mathrm{em}}(z)
$$

\footnotetext{
${ }^{1}$ The event has been posted on Aug. 14, 2019, about six weeks after the v1 version of this paper appeared on the arxiv.
} 
The value of $z$ inferred from a measurement of $d_{L}(z)$ depends in particular on the value chosen for $H_{0}$. Given the well-known discrepancy, mentioned above, between the value obtained from the local measurement of $H_{0}$ and that from Planck (combined with $\mathrm{BAO}$ and $\mathrm{SNe}$ ), this gives a systematic error that is larger than the statistical error of each measurement, so the two cases must be treated separately. We first assume the correctness of the value of $H_{0}$ obtained from Planck+BAO+SNe. Then for $\Lambda \mathrm{CDM}$ we use the values $H_{0}=67.60_{-0.55}^{+0.66}$ (in units of $\mathrm{km} \mathrm{s}^{-1} \mathrm{Mpc}^{-1}$ ) and $\Omega_{M}=0.3109$ from the first column of Table $1 .^{2}$ In this case we find that $d_{L}=(267 \pm 52) \mathrm{Mpc}$ corresponds to a source redshift

$$
z=0.058 \pm 0.011, \quad\left(\Lambda \mathrm{CDM}, H_{0} \text { from Planck+BAO+SNe }\right)
$$

where the error quoted is the one induced by the error on the measurement of $d_{L}$. The statistical error on the measurement of $H_{0}$ induces a further error $\Delta z=z\left(\Delta H_{0}\right) / H_{0} \simeq 0.0005$, which is beyond the number of digits given in eq. (3.4). Let us compare this result with that obtained in the RT model, using $\Delta N=64$ to maximize the effect of modified GW propagation. We then use the mean values for $H_{0}$ and $\Omega_{M}$ obtained for the RT model (with $\Delta N=64)$ from the Planck+BAO+SNe observations, given in the last column of Table 1. Using eqs. (3.2) and (3.3) and inverting $d_{L}^{\mathrm{gw}}(z)=(267 \pm 52) \mathrm{Mpc}$, we find that the redshift of the source is

$$
z=0.054 \pm 0.010, \quad\left(\mathrm{RT} \text { with } \Delta N=64, H_{0} \text { from Planck+BAO+SNe }\right)
$$

and the actual ('electromagnetic') luminosity distance of the source is $d_{L}^{\mathrm{em}}=(251 \pm 49) \mathrm{Mpc}$.

If instead we use the value $H_{0}=74.03 \pm 1.42$ from local measurements [1], in $\Lambda$ CDM we get

$$
z=0.063 \pm 0.012, \quad\left(\Lambda \mathrm{CDM}, H_{0}=74.03\right),
$$

while the RT model predicts that the redshift of the source is rather

$$
z=0.059 \pm 0.010, \quad\left(\text { RT with } \Delta N=64, H_{0}=74.03\right)
$$

and its electromagnetic luminosity distance is $d_{L}^{\mathrm{em}}=(250 \pm 49) \mathrm{Mpc}$. To both eqs. (3.6) and (3.7) one should add $\Delta z=z\left(\Delta H_{0}\right) / H_{0} \simeq 0.001$ from the error on $H_{0}$. In both cases ( $H_{0}$ from Planck+BAO+SNe, or $H_{0}$ from local measurements) the RT model with $\Delta N=64$ predicts that the actual cosmological redshift of the source is smaller than the value that would be inferred using $\Lambda \mathrm{CDM}$, by about $7 \%$. This prediction can in principle be tested if an electromagnetic counterpart will be discovered. Of course, to test this prediction one must first be able to arbitrate the tension between Planck and local measurements of $H_{0}$, which can be done with standard sirens themselves. However, it is quite remarkable that, in the RT model, even for a single event at the distance of S190814bv, the effect from modified GW propagation on the redshift, or equivalently on the luminosity distance, can be as large as $7 \%$ (with this value reached for $\Delta N=64$, and smaller effects for smaller $\Delta N$ ), which is well above the statistical errors of both the Planck and the local measurements of $H_{0}$.

Acknowledgments. The work of E.B., A.F., S.F. and M.M. is supported by the Swiss National Science Foundation and by the SwissMap National Center for Competence in Research. The work of Y.D. is supported by Swiss National Science Foundation and by a Consolidator Grant of the European Research Council (ERC-2015-CoG grant 680886).

\footnotetext{
${ }^{2}$ Note that, since we will get $z \simeq 0.06$, we are in the regime where $d_{L} \simeq H_{0}^{-1} z[1+\mathcal{O}(z)]$, so the precise value of $\Omega_{M}$ has limited impact, and the error on $\Omega_{M}$ has no influence on the error on $z$.
} 


\section{References}

[1] A. G. Riess, S. Casertano, W. Yuan, L. M. Macri, and D. Scolnic, "Large Magellanic Cloud Cepheid Standards Provide a 1\% Foundation for the Determination of the Hubble Constant and Stronger Evidence for Physics Beyond LambdaCDM," Astrophys. J. 876 no. 1, (2019) 85, arXiv: 1903.07603 [astro-ph.CO].

[2] Planck Collaboration, P. A. R. Ade et al., "Planck 2015 results. XIII. Cosmological parameters," Astron. Astrophys. 594 (2016) A13, arXiv:1502.01589 [astro-ph.C0].

[3] DES Collaboration, T. M. C. Abbott et al., "Dark Energy Survey Year 1 Results: Constraints on Extended Cosmological Models from Galaxy Clustering and Weak Lensing," Phys. Rev. D99 (2019) 123505, arXiv:1810.02499 [astro-ph.C0].

[4] B. P. Abbott et al., "Observation of Gravitational Waves from a Binary Black Hole Merger," Phys. Rev. Lett. 116 (2016) 061102, arXiv:1602.03837 [gr-qc].

[5] B. Abbott et al., "GW170817: Observation of Gravitational Waves from a Binary Neutron Star Inspiral," Phys. Rev. Lett. 119 (2017) 161101, arXiv:1710.05832 [gr-qc].

[6] B. P. Abbott et al., "GWTC-1: A Gravitational-Wave Transient Catalog of Compact Binary Mergers Observed by LIGO and Virgo during the First and Second Observing Runs," arXiv:1811.12907 [astro-ph.HE].

[7] B. P. Abbott et al., "Gravitational Waves and Gamma-rays from a Binary Neutron Star Merger: GW170817 and GRB 170817A," Astrophys. J. 848 (2017) L13, arXiv:1710.05834 [astro-ph.HE].

[8] P. Creminelli and F. Vernizzi, "Dark Energy after GW170817 and GRB170817A," Phys. Rev. Lett. 119 (2017) 251302, arXiv:1710.05877 [astro-ph.CO].

[9] J. Sakstein and B. Jain, "Implications of the Neutron Star Merger GW170817 for Cosmological Scalar-Tensor Theories," Phys. Rev. Lett. 119 (2017) 251303, arXiv:1710.05893 [astro-ph.CO].

[10] J. M. Ezquiaga and M. Zumalacárregui, "Dark Energy After GW170817: Dead Ends and the Road Ahead," Phys. Rev. Lett. 119 (2017) 251304, arXiv:1710.05901 [astro-ph.Co].

[11] T. Baker, E. Bellini, P. G. Ferreira, M. Lagos, J. Noller, and I. Sawicki, "Strong constraints on cosmological gravity from GW170817 and GRB 170817A," Phys. Rev. Lett. 119 (2017) 251301, arXiv:1710.06394 [astro-ph.C0].

[12] I. D. Saltas, I. Sawicki, L. Amendola, and M. Kunz, "Anisotropic Stress as a Signature of Nonstandard Propagation of Gravitational Waves," Phys. Rev. Lett. 113 (2014) 191101, arXiv:1406.7139 [astro-ph.CO].

[13] J. Gleyzes, D. Langlois, and F. Vernizzi, "A unifying description of dark energy," Int. J. Mod. Phys. D23 (2014) 1443010, arXiv:1411.3712 [hep-th].

[14] L. Lombriser and A. Taylor, "Breaking a Dark Degeneracy with Gravitational Waves," JCAP 1603 (2016) 031, arXiv:1509.08458 [astro-ph.CO].

[15] A. Nishizawa, "Generalized framework for testing gravity with gravitational-wave propagation. I. Formulation," Phys. Rev. D97 (2018) 104037, arXiv:1710.04825 [gr-qc].

[16] S. Arai and A. Nishizawa, "Generalized framework for testing gravity with gravitational-wave propagation. II. Constraints on Horndeski theory," Phys. Rev. D97 (2018) 104038, arXiv: 1711.03776 [gr-qc].

[17] E. Belgacem, Y. Dirian, S. Foffa, and M. Maggiore, "The gravitational-wave luminosity distance in modified gravity theories," Phys. Rev. D97 (2018) 104066, arXiv:1712.08108 [astro-ph.CO]. 
[18] L. Amendola, I. Sawicki, M. Kunz, and I. D. Saltas, "Direct detection of gravitational waves can measure the time variation of the Planck mass," JCAP 1808 (2018) 030, arXiv:1712.08623 [astro-ph.CO].

[19] E. Belgacem, Y. Dirian, S. Foffa, and M. Maggiore, "Modified gravitational-wave propagation and standard sirens," Phys. Rev. D98 (2018) 023510, arXiv:1805.08731 [gr-qc].

[20] E. V. Linder, "No Slip Gravity," JCAP 1803 (2018) 005, arXiv:1801.01503 [astro-ph.Co].

[21] M. Lagos, M. Fishbach, P. Landry, and D. E. Holz, "Standard sirens with a running Planck mass," Phys. Rev. D99 (2019) 083504, arXiv:1901.03321 [astro-ph.C0].

[22] A. Nishizawa and S. Arai, "Generalized framework for testing gravity with gravitational-wave propagation. III. Future prospect," Phys. Rev. D99 (2019) 104038, arXiv:1901.08249 [gr-qc].

[23] LISA Cosmology Working Group Collaboration, E. Belgacem et al., "Testing modified gravity at cosmological distances with LISA standard sirens," JCAP 1907 (2019) 024, arXiv:1906.01593 [astro-ph.CO].

[24] C. Deffayet and K. Menou, "Probing Gravity with Spacetime Sirens," Astrophys. J. 668 (2007) L143-L146, arXiv:0709.0003 [astro-ph].

[25] K. Pardo, M. Fishbach, D. E. Holz, and D. N. Spergel, "Limits on the number of spacetime dimensions from GW170817," JCAP 1807 (2018) 048, arXiv:1801.08160 [gr-qc].

[26] E. Belgacem, Y. Dirian, S. Foffa, E. J. Howell, M. Maggiore, and T. Regimbau, "Cosmology and dark energy from joint gravitational wave-GRB observations," JCAP, to appear (2019), arXiv:1907.01487 [astro-ph.CO].

[27] T. Taylor and G. Veneziano, "Quantum Gravity at Large Distances and the Cosmological Constant," Nucl.Phys. B345 (1990) 210-230.

[28] I. Antoniadis and E. Mottola, "Graviton Fluctuations in De Sitter Space," J.Math.Phys. 32 (1991) 1037-1044.

[29] I. Antoniadis and E. Mottola, "4-D quantum gravity in the conformal sector," Phys.Rev. D45 (1992) 2013-2025.

[30] N. C. Tsamis and R. P. Woodard, "Strong infrared effects in quantum gravity," Annals Phys. 238 (1995) 1-82.

[31] A. Polyakov, "De Sitter space and eternity," Nucl.Phys. B797 (2008) 199-217, arXiv:0709.2899 [hep-th].

[32] S. Deser and R. Woodard, "Nonlocal Cosmology," Phys.Rev.Lett. 99 (2007) 111301, arXiv:0706.2151 [astro-ph].

[33] C. Wetterich, "Effective nonlocal Euclidean gravity," Gen.Rel.Grav. 30 (1998) 159-172, arXiv:gr-qc/9704052 [gr-qc].

[34] E. Belgacem, A. Finke, A. Frassino, and M. Maggiore, "Testing nonlocal gravity with Lunar Laser Ranging," JCAP 1902 (2019) 035, arXiv:1812.11181 [gr-qc].

[35] M. Maggiore, "Phantom dark energy from nonlocal infrared modifications of general relativity," Phys.Rev. D89 (2014) 043008, arXiv: 1307.3898 [hep-th].

[36] N. Arkani-Hamed, S. Dimopoulos, G. Dvali, and G. Gabadadze, "Nonlocal modification of gravity and the cosmological constant problem," arXiv:hep-th/0209227 [hep-th].

[37] M. Jaccard, M. Maggiore, and E. Mitsou, "A non-local theory of massive gravity," Phys.Rev. D88 (2013) 044033, arXiv:1305.3034 [hep-th].

[38] M. Maggiore, "Nonlocal Infrared Modifications of Gravity. A Review," Fundam. Theor. Phys. 187 (2017) 221-281, arXiv:1606.08784 [hep-th]. 
[39] E. Belgacem, Y. Dirian, S. Foffa, and M. Maggiore, "Nonlocal gravity. Conceptual aspects and cosmological predictions," JCAP 1803 (2018) 002, arXiv:1712.07066 [hep-th].

[40] S. Foffa, M. Maggiore, and E. Mitsou, "Cosmological dynamics and dark energy from non-local infrared modifications of gravity," Int.J.Mod.Phys. A29 (2014) 1450116, arXiv:1311.3435 [hep-th].

[41] A. Kehagias and M. Maggiore, "Spherically symmetric static solutions in a non-local infrared modification of General Relativity," JHEP 1408 (2014) 029, arXiv:1401.8289 [hep-th].

[42] M. Maggiore and M. Mancarella, "Non-local gravity and dark energy," Phys.Rev. D90 (2014) 023005, arXiv: 1402.0448 [hep-th].

[43] S. Nesseris and S. Tsujikawa, "Cosmological perturbations and observational constraints on nonlocal massive gravity," Phys.Rev. D90 (2014) 024070, arXiv:1402.4613 [astro-ph.Co].

[44] Y. Dirian, S. Foffa, N. Khosravi, M. Kunz, and M. Maggiore, "Cosmological perturbations and structure formation in nonlocal infrared modifications of general relativity," JCAP 1406 (2014) 033, arXiv:1403.6068 [astro-ph.C0].

[45] A. Barreira, B. Li, W. A. Hellwing, C. M. Baugh, and S. Pascoli, "Nonlinear structure formation in Nonlocal Gravity," JCAP 1409 (2014) 031, arXiv:1408.1084 [astro-ph.C0].

[46] Y. Dirian, S. Foffa, M. Kunz, M. Maggiore, and V. Pettorino, "Non-local gravity and comparison with observational datasets," JCAP 1504 (2015) 044, arXiv: 1411.7692 [astro-ph.CO].

[47] Y. Dirian, S. Foffa, M. Kunz, M. Maggiore, and V. Pettorino, "Non-local gravity and comparison with observational datasets. II. Updated results and Bayesian model comparison with $\Lambda$ CDM," JCAP 1605 (2016) 068, arXiv:1602.03558 [astro-ph.C0].

[48] Y. Dirian, "Changing the Bayesian prior: Absolute neutrino mass constraints in nonlocal gravity," Phys. Rev. D96 (2017) 083513, arXiv:1704.04075 [astro-ph.Co].

[49] E. Belgacem, G. Cusin, S. Foffa, M. Maggiore, and M. Mancarella, "Stability issues of nonlocal gravity during primordial inflation," Int. J. Mod. Phys. A33 (2018) 1850007, arXiv:1610.05664 [hep-th]. [Erratum: Int. J. Mod. Phys.A33,1892005(2018)].

[50] V. Poulin, K. K. Boddy, S. Bird, and M. Kamionkowski, "Implications of an extended dark energy cosmology with massive neutrinos for cosmological tensions," Phys. Rev. D97 (2018) 123504, arXiv:1803.02474 [astro-ph.C0].

[51] K. Aylor, M. Joy, L. Knox, M. Millea, S. Raghunathan, and W. L. K. Wu, "Sounds Discordant: Classical Distance Ladder \& $\Lambda$ CDM -based Determinations of the Cosmological Sound Horizon," Astrophys. J. 874 (2019) 4, arXiv:1811.00537 [astro-ph.CO]. 